\title{
On special submanifolds of the Page space
}

\author{
Mustafa Kalafat Ramazan Sar
}

September 4, 2021

\begin{abstract}
In this paper, we study some classes of submanifolds of codimension one and two in the Page space. These submanifolds are totally geodesic. We also compute their curvature and show that some of them are constant curvature spaces. Finally we give information on how the Page space is related to some other metrics on the same underlying smooth manifold.
\end{abstract}

\section{Introduction}

Let $M$ be a complex manifold with complex structure $J$. We say that a Riemannian metric $g$ on a complex manifold $M$ is Hermitian if $|X|_{g}=|J X|_{g}$ for any $X \in T_{x} M$ and $x \in M$. Then the triplet $(M, J, g)$ is called Hermitian manifold. A Riemannian metric is called Einstein if its Ricci tensor Ric is a constant multiple of itself, i.e. Ric $=\lambda g$ for same constant $\lambda \in \mathbb{R}$ called the Einstein constant. If the metric is Einstein on the Hermitian manifold $M$, then we call it Einstein-Hermitian. In [Pag78], D. Page introduced an Einstein-Hermitian metric on the blow up of the complex projective plane. The manifold is the nontrivial $S^{2}$ fibre bundle over $S^{2}$ and has $\chi=4$ and $\tau=0$. It is the unique Einstein-Hermitian non Kähler metric on the blow up of complex projective plane. One can first think of the following metric on the product $S^{3} \times I$ where $I$ is the closed interval $[0, \pi]$

$$
g_{\text {Page }}:=V(r) d r^{2}+f(r)\left(\sigma_{1}^{2}+\sigma_{2}^{2}\right)+\frac{C \sin ^{2} r}{V(r)} \sigma_{3}^{2}
$$

where the functions

$$
\begin{aligned}
V(r) & =\frac{1-a^{2} \cos ^{2} r}{3-a^{2}-a^{2}\left(1+a^{2}\right) \cos ^{2} r} \\
f(r) & =4 \frac{1-a^{2} \cos ^{2} r}{3+6 a^{2}-a^{4}} \\
C & =\left(\frac{2}{3+a^{2}}\right)^{2}
\end{aligned}
$$


$\sigma_{1}, \sigma_{2}, \sigma_{3}$ are standard left invariant 1 -forms on the Lie group $S U(2) \approx S^{3}$ and $a$ is a small, positive constant which can approximately be computed as $a \approx 0.28170$ (So $C \approx 0.42183$ ). It is actually defined to be the unique positive root of the following quartic polynomial,

$$
a^{4}+4 a^{3}-6 a^{2}+12 a-3=0 .
$$

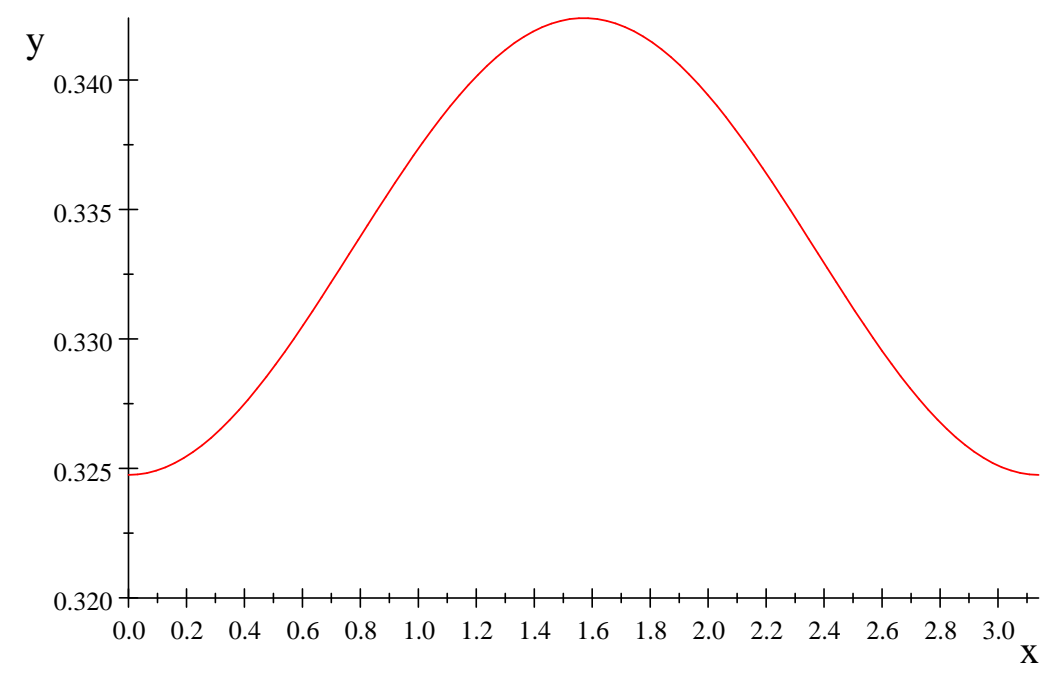

Figure 1: The function $V(r)$ for $r \in[0, \pi]$. Minimum values are $V(0, \pi) \approx 0.324776$ and the maximum value $V(\pi / 2) \approx 0.342397$.

At the end points $r=0$ and $r=\pi$, the metric shrinks to the the round metric on $S^{2}$. Thus, $g$ descends to the metric $g_{\text {Page }}$ on the quotient $\left(S^{3} \times I\right) / \sim$ where $\sim$ identifies the fibers of the Hopf fibration $h: S^{3} \rightarrow S^{2}$ on the two ends $S^{3} \times\{0\}$ and $S^{3} \times\{\pi\}$ of the cylinder $S^{3} \times I$. The resulting manifold is indeed the connected sum $\mathbb{C P}_{2} \sharp \overline{\mathbb{C P}}_{2}$. See $\left[\mathrm{KK}_{18}\right]$ for a mathematical exposition of the Page metric for further details. There, an efficient coordinate system is introduced and studied. This coordinate system uses Euler angles on the $S^{3} \subset \mathbb{R}^{4}$ which e.g. realize the Hopf fibration in the best.

$$
0 \leq \theta \leq \pi, \quad 0 \leq \phi \leq 2 \pi, \quad 0 \leq \psi \leq 4 \pi
$$




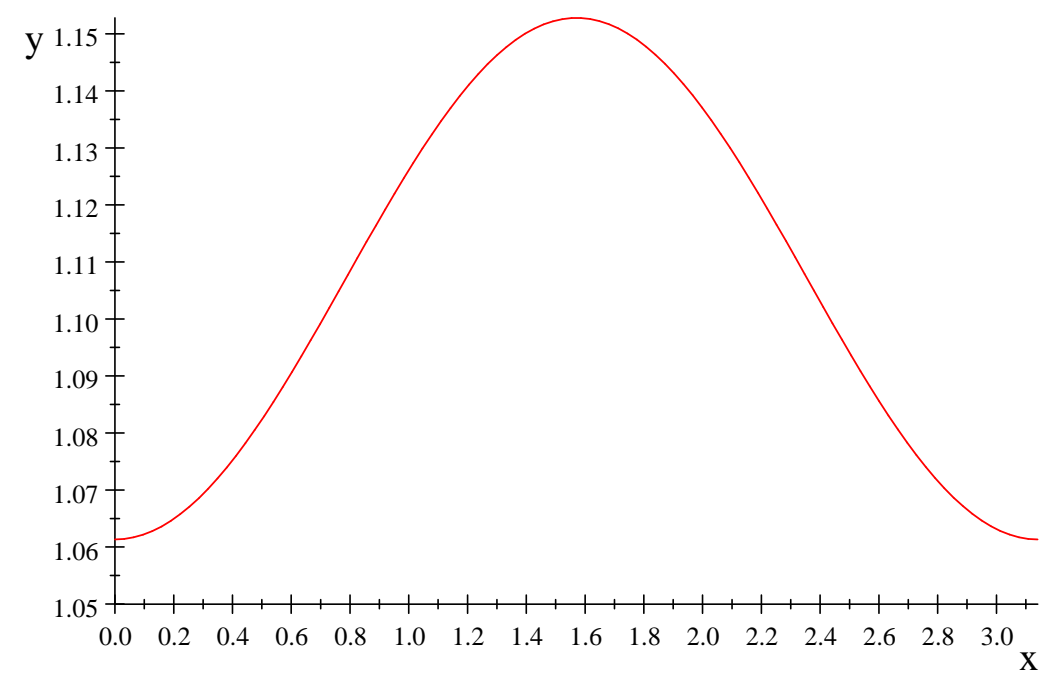

Figure 2: The function $f(r)$ for $r \in[0, \pi]$. Here, at the endpoints $f(0)=f(\pi) \approx$ 1.061462 , the maximum value is approximately equal to 1.152811 , taken in the middle.

$$
\begin{aligned}
& x_{1}:=r \cos \frac{\theta}{2} \cos \frac{\psi+\phi}{2} \\
& x_{2}:=r \cos \frac{\theta}{2} \sin \frac{\psi+\phi}{2} \\
& x_{3}:=r \sin \frac{\theta}{2} \cos \frac{\psi-\phi}{2} \\
& x_{0}:=r \sin \frac{\theta}{2} \sin \frac{\psi-\phi}{2}
\end{aligned}
$$

where the Hopf fibration in these coordinates is just a projection [FIPo4],

$$
h: S^{3} \rightarrow S^{2}, \quad h(\theta, \psi, \phi)=(-\phi, \theta) .
$$

Here the exchange $\phi \leftrightarrow \theta$ is needed to relate to the calculus angles on $S^{2}$. Changing $\psi$ does not change the element in the image. So, whenever the image $\phi, \theta$ is fixed, $\psi$ parametrizes the Hopf circle. An invariant coframe on $S^{3}$ is given as follows:

$$
\begin{aligned}
& \sigma_{1}=(\sin \psi d \theta-\sin \theta \cos \psi d \phi) / 2 \\
& \sigma_{2}=(-\cos \psi d \theta-\sin \theta \sin \psi d \phi) / 2 \\
& \sigma_{3}=(d \psi+\cos \theta d \phi) / 2
\end{aligned}
$$

In terms of these new coordinates the metric becomes, 


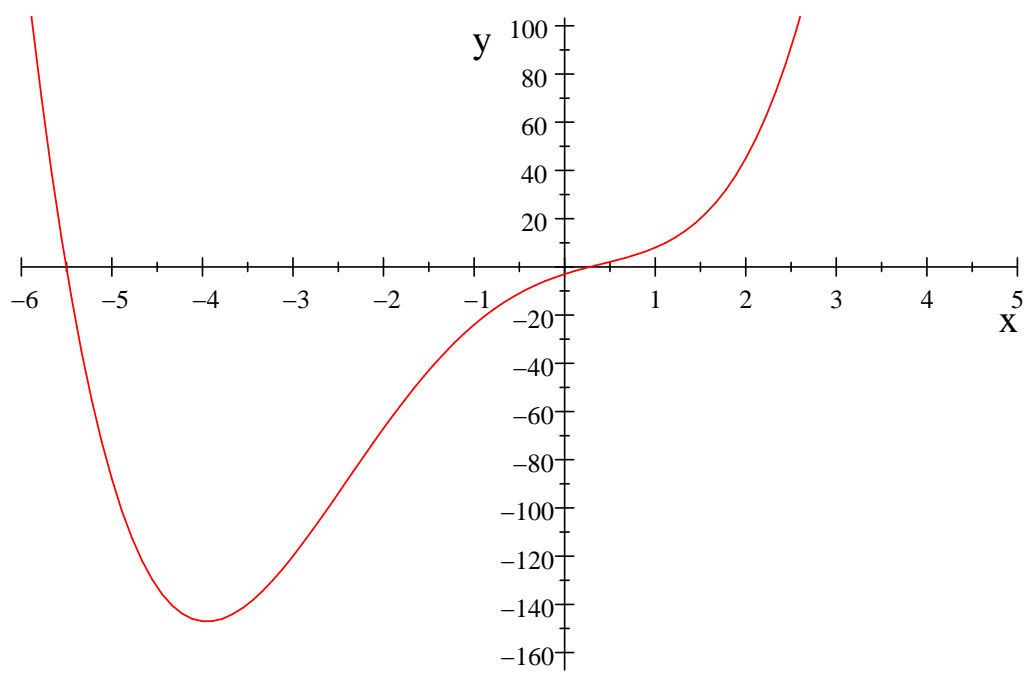

Figure 3: The polynomial $p(x)=x^{4}+4 x^{3}-6 x^{2}+12 x-3$.

$g_{\text {Page }}=V d r^{2}+\left\{\frac{f}{4} \sin ^{2} \theta+\frac{C \sin ^{2} r \cos ^{2} \theta}{4 V(r)}\right\} d \phi^{2}+\frac{C \sin ^{2} r}{4 V(r)} d \psi^{2}+\frac{C \sin ^{2} r \cos \theta}{4 V(r)}(d \psi \otimes d \phi+d \phi \otimes d \psi)+\frac{f}{4} d \theta^{2}$.

Letting $U:=\sqrt{V(r)}, h:=\sqrt{f(r)}, D:=\sqrt{C}$ we choose the simplest Vierbein i.e. orthonormal coframe as follows

$$
\left\{e^{0}, e^{1}, e^{2}, e^{3}\right\}:=\left\{U d r, 2^{-1} h \sin \theta d \phi, 2^{-1} U^{-1} D \sin r(d \psi+\cos \theta d \phi), 2^{-1} h d \theta\right\} .
$$

After this, using the structure equations [EGH80] $d e^{a}=-\omega^{a}{ }_{b} \wedge e^{b}$ for $0 \leq a \leq 3$, connection 1-forms of the Page metric can be computed as the following.

$$
\begin{array}{r}
\omega_{0}^{1}=U^{-1} h^{-1} \dot{h} e^{1}+2^{-1} U^{-1} h^{-1} \dot{h} \sin ^{-1} \theta e^{3}, \omega_{0}{ }_{0}=\sin ^{-1} r\left(U^{-1} \sin r\right)_{r} e^{2}, \omega_{0}^{3}=2^{-1} U^{-1} h^{-1} \dot{h} \sin ^{-1} \theta e^{1} \\
\omega^{2}{ }_{1}=D U^{-1} h^{-2} \sin r e^{3}, \omega_{3}^{1}=2^{-1} U^{-1} h^{-1} \dot{h} \sin ^{-1} \theta e^{0}+2 h^{-1} \cot \theta e^{1}-D U^{-1} h^{-2} \sin r e^{2} \\
\omega^{3}{ }_{2}=D U^{-1} h^{-2} \sin r e^{1}
\end{array}
$$

See Appendix A for details. Note that these 1-forms constitute a skew-symmetric matrix of forms. One can further go and compute the curvature 2-forms using the identity $R_{b}^{a}:=d \omega^{a}{ }_{b}+\omega^{a}{ }_{c} \wedge \omega^{c}{ }_{b}$. These make up a skew-symmetric matrix of 2-forms as well. Looking at the coefficients with respect to a basis yields the Riemann curvature tensor coefficients $R^{a}{ }_{b}=\frac{1}{2} R^{a}{ }_{b c d} e^{c} \wedge e^{d}$. 
We summarize some of our results as follows.

Theorem 1.1. There are totally geodesic subsurfaces of the Page space which are isometric to tori and sphere with their constant zero and positive curvature metrics respectively.

Theorem 1.2. There are totally geodesic hypersurfaces of the Page space which are diffeomorphic to $S^{3}$ and $S^{1} \times S^{2}$.

In sections $\$ 2$ and $\$ 3$ we study the surfaces and hypersurfaces inside the Page space. In \$4 we compare it with the other metrics and study the relation with the Yamabe problem. Finally in Appendix $\$ \mathrm{~A}$ we derive the connection 1-forms.

Acknowledgements. Thanks to C. Koca and Ö. Kelekçi for useful comments. This work is partially supported by Tübitak (Turkish science and research council) grant $\sharp_{113} F_{159}$. The Ansi $C$ programing language [KR88] is used for the computation of some min/max results. Thanks to Alphan Es for helping with the graphics.

\section{Minimal surfaces inside the Page Space and their curvature}

Now we are in a position to analyse some of the submanifolds with partially constant Euler coordinates. Here, keep in mind that $\psi$ is the Hopf circle direction.

$\mathbf{S}_{1}$ : By keeping $r \in(0, \pi), \theta \in[0, \pi]$ fixed and varying $\psi, \phi$ one obtains tori. At the ends for $r=0, \pi$, this construction produces circles. The restriction of the metric to these tori is computed as

$$
g_{S_{1}}=\left\{\frac{f}{4} \sin ^{2} \theta+\frac{C \sin ^{2} r \cos ^{2} \theta}{4 V(r)}\right\} d \phi^{2}+\frac{C \sin ^{2} r}{4 V(r)} d \psi^{2}+\frac{C \sin ^{2} r \cos ^{2} \theta}{4 V(r)}(d \psi \otimes d \phi+d \phi \otimes d \psi) .
$$

We can work with the orthonormal coframe $\left\{e^{1}, e^{2}\right\}$ selecting from (1.4) on these surfaces since this spans the $\phi \psi$ plane. Connection 1-form can be computed from the structure equations $0=d e^{i}+\widetilde{\omega}^{i}{ }_{j} \wedge e^{j}$. Both components vanish hence $\widetilde{\omega}_{2}^{1}=0$.

$$
\begin{gathered}
\widetilde{R}_{2}^{1}=d \widetilde{\omega}^{1}{ }_{2}+\widetilde{\omega}^{1} \wedge \widetilde{\omega}^{i}{ }_{2}=0 . \\
\widetilde{R}_{2}^{1}=\frac{1}{2} \widetilde{R}_{212}^{1} e^{1} \wedge e^{2} \text { implies that } \widetilde{R}_{1212}=\widetilde{R}_{212}^{1}=0 .
\end{gathered}
$$

So that all these tori are flat. We know that the ambient connection $\left.\omega^{1}{ }_{2}\right|_{S_{1}}=0$ so that the second fundamental form, which is the difference of two connections, vanishes on these subtori. Hence these tori are totally geodesic, consequently minimal. One can alternatively compute Christoffel symbols from metric coefficients 
directly to see that they all vanish to yield zero curvature. The metric is conformally equivalent to

$$
\begin{gathered}
\widetilde{g}_{S_{1}}=\left\{C^{-1} f V \sin ^{-2} r \sin ^{2} \theta+\cos ^{2} \theta\right\} d \phi^{2}+d \psi^{2}+\cos ^{2} \theta(d \psi \otimes d \phi+d \phi \otimes d \psi) . \\
\widetilde{g}_{i j}=\left[\begin{array}{cc}
C^{-1} f V \sin ^{-2} r \tan ^{2} \theta+1 & 1 \\
1 & \cos ^{-2} \theta
\end{array}\right]
\end{gathered}
$$

We can compute the angle of the parallelogram as follows.

$$
\cos \Theta=g_{\phi \psi} / \sqrt{g_{\phi \phi} g_{\psi \psi}}=\cos \theta / \sqrt{C^{-1} f V \sin ^{-2} r \tan ^{2} \theta+1}
$$

The radius function is the following,

$$
\mathrm{R}=\int_{0}^{2 \pi}\left|\partial_{\phi}\right| d \phi / \int_{0}^{4 \pi}\left|\partial_{\psi}\right| d \psi=2^{-1} g_{\phi \phi} / g_{\psi \psi}=2^{-1}\left\{C^{-1} f V \sin ^{-2} r \sin ^{2} \theta+\cos ^{2} \theta\right\} .
$$

If we compute he minimum and maximum of the radius and cosine while varying $r \in(0, \pi), \theta \in[0, \pi]$, we got the following intervals,

$$
R \in[0.408520, \infty) \text { and } \cos \Theta \in[-1,1] \text {. }
$$

where the minimum radius is taken at $r=1.571425, \theta=1.571425$. See Figure 4 for the coverage of these metrics on the Teichmüller space. Note that the whole moduli space is covered with these classes of flat metrics.

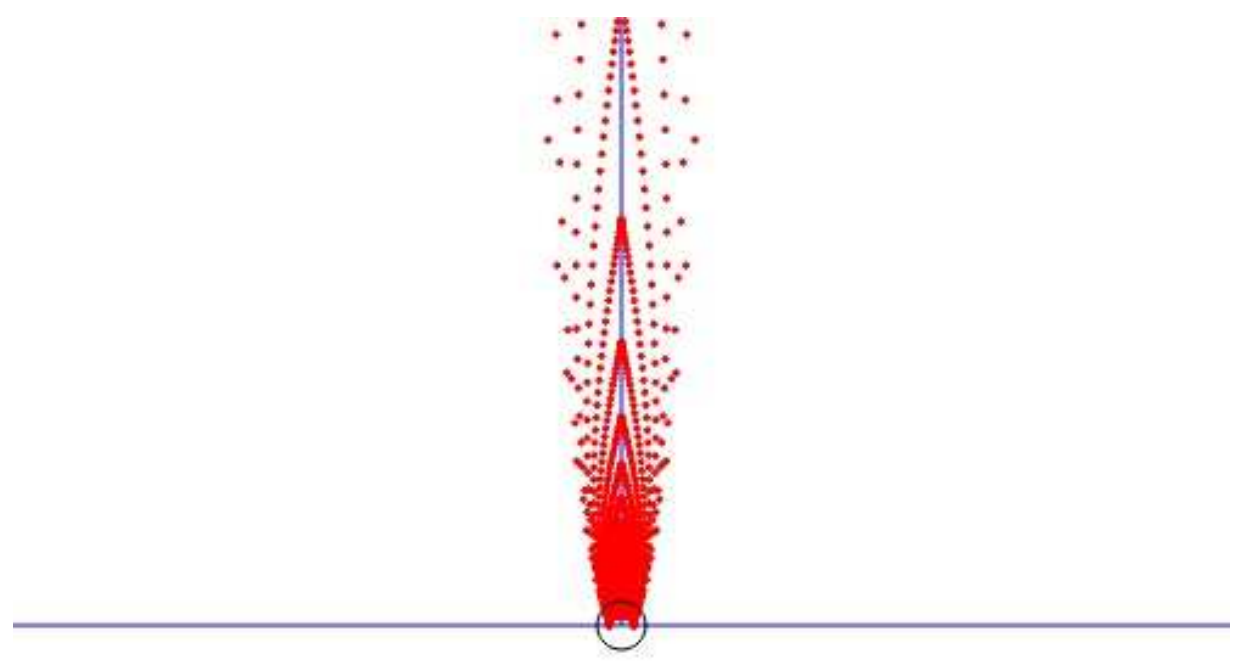

Figure 4: Image of the $\psi, \phi$ tori $S_{1}$ on the Teichmüller space. 
$\mathbf{S}_{\mathbf{2}}$ : This time keep $\psi, \phi$ fixed and vary $r, \theta$ to obtain a cylinder. Its two ends lie on the two Hopf spheres. Then, plugging these into the Page metric's expression we get

$$
g_{S_{2}}=V d r^{2}+\frac{f}{4} d \theta^{2}
$$

and using the orthonormal coframe $\left\{e^{0}, e^{3}\right\}$ from the two structure equations we get,

$$
\begin{aligned}
& 0=d e^{0}+\widetilde{\omega}_{3}^{0} \wedge e^{3}=0+\widetilde{\omega}_{3}^{0} \wedge e^{3} \\
& 0=d e^{3}+\widetilde{\omega}_{0}^{3} \wedge e^{0}=2^{-1} \dot{h} d r \wedge d \theta+\widetilde{\omega}_{0}^{3} \wedge U d r
\end{aligned}
$$

we conclude that $\quad \widetilde{\omega}_{0}^{3}=2^{-1} U^{-1} h d \theta=U^{-1} h^{-1} \dot{h} e^{3}$, which is the same as the one in the ambient space so that these cylinders are totally geodesic. Computing the curvature 2-form,

$$
\begin{aligned}
\widetilde{R}_{3}^{0} & =d \widetilde{\omega}_{3}^{0}+\widetilde{\omega}_{i}^{0} \wedge \widetilde{\omega}^{i}{ }_{3} \\
& =2^{-1}\left(U^{-2} \dot{U} \dot{h}-U^{-1} \dot{h}\right) d r \wedge d \theta \\
& =\left(U^{-3} \dot{U}-U^{-2} h^{-1} \dot{h}\right) e^{0} \wedge e^{3} .
\end{aligned}
$$

So that the Gaussian curvature is equal to $\widetilde{R}_{0303}=\widetilde{R}_{303}^{0}=U^{-3} h^{-1} \dot{U} \dot{h}-$ $U^{-2} h^{-1} \dot{h}$.

$\mathbf{S}_{3}$ : We keep $r, \phi$ fixed and vary $\psi, \theta$ to obtain an embedded cylinder. If we pass beyond the chart and vary $\theta \in[0,2 \pi]$, then we get a torus. At the endpoints for $r=0, \pi$ these tori degenerate to a circle parametrized by the angle $\theta$. Metric on the tori is a product, so that these tori are flat. If we conformally rescale its metric we get,

$$
\widetilde{g}_{S_{3}}=\frac{C \sin ^{2} r}{f(r) V(r)} d \psi^{2}+d \theta^{2}
$$

The graph of the coefficient function can be seen in Figure 5. The range of this function is approximately in the open interval $(0,1.068802)$ for $r \in(0, \pi)$. So that if one looks at the image of these tori on the Teichmüller space [Joso6] that is a line on the imaginary axis of height slightly above $i$. The part under $i$ can be carried onto the moduli space via the linear fractional transformation $f(z)=-1 / z \in$ $\operatorname{PSL}(2, \mathbb{Z})$. So that it covers the whole imaginary axis above $i$. See Figure 6 ,

$\mathbf{S}_{4}$ : We keep $\theta, \psi$ fixed and vary $r, \phi$ to obtain a cylinder with boundary components on the Hopf spheres at the two ends. Metric on this cylinder is

$$
g_{S_{4}}=V d r^{2}+\left\{\frac{f}{4} \sin ^{2} \theta+\frac{C \sin ^{2} r \cos ^{2} \theta}{4 V(r)}\right\} d \phi^{2}
$$

and orthonormal frame $\left\{e^{0}, e^{1}\right\}$, from the two structure equations, 


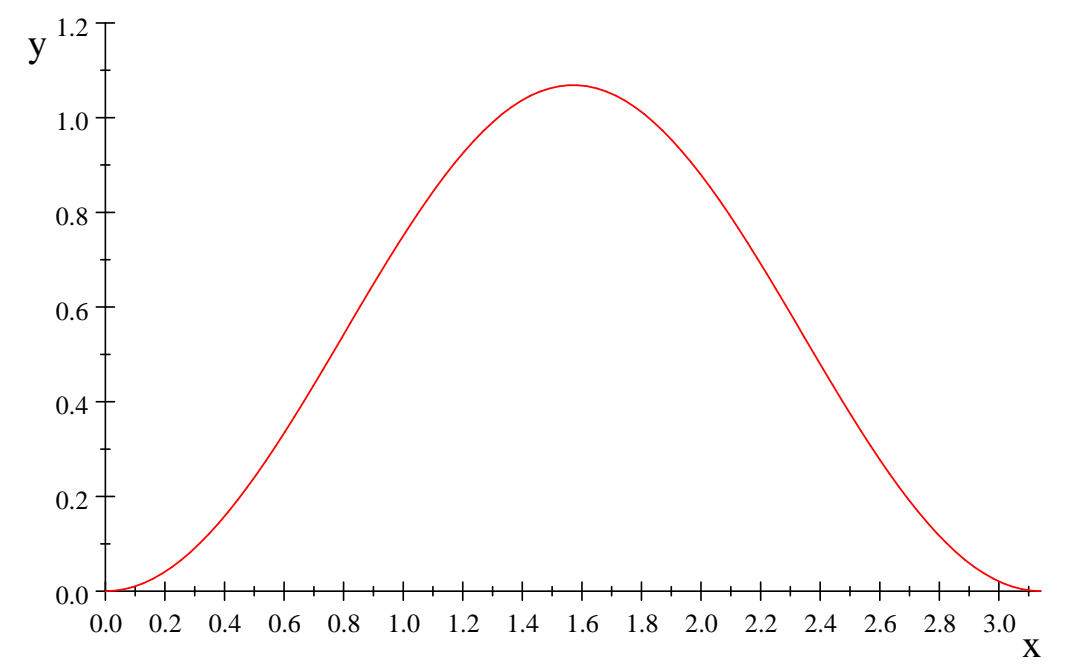

Figure 5: The coefficient function $C \sin ^{2} r / f V$ of the conformally rescaled metric on the torus for $r \in[0, \pi]$. This function vanishes at the endpoints and takes the approximate maximum value 1.068802 at the midpoint.

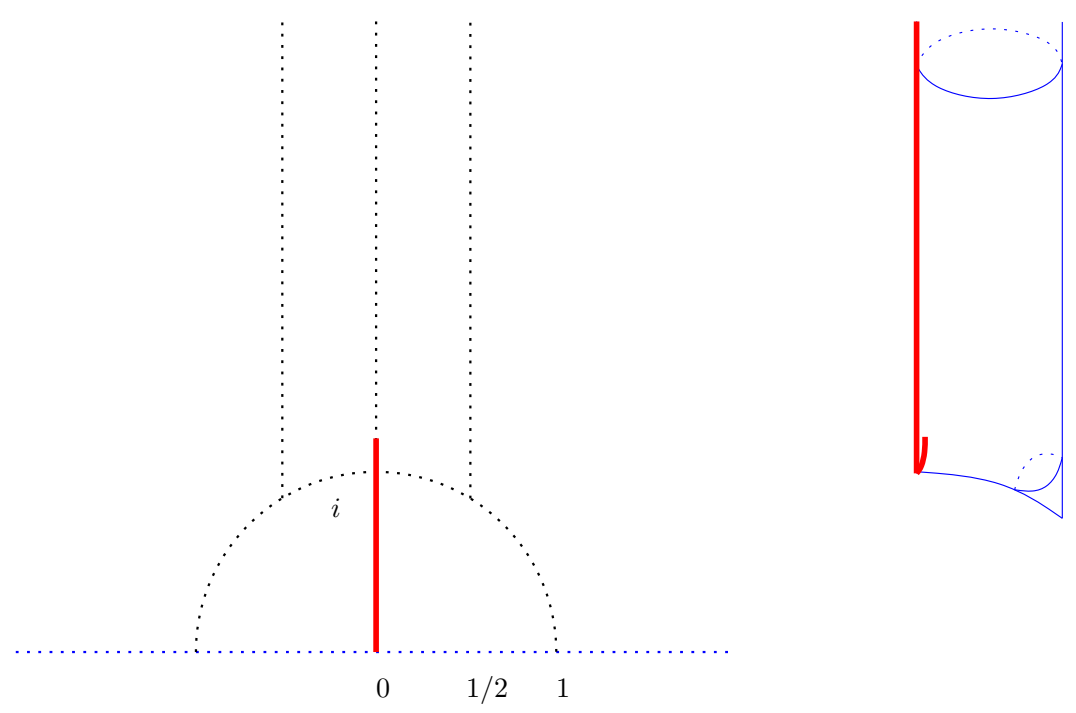

Figure 6: Image of the $\psi, \theta$ tori $S_{3}$ on the Teichmüller and Moduli space.

$$
\begin{aligned}
& 0=d e^{0}+\widetilde{\omega}^{0}{ }_{1} \wedge e^{1}=\widetilde{\omega}^{0}{ }_{1} \wedge e^{1} \\
& 0=d e^{1}+\widetilde{\omega}_{0}^{1} \wedge e^{0}=2^{-1} \dot{h} \sin \theta d r \wedge d \phi+\widetilde{\omega}_{0}{ }_{0} \wedge U d r
\end{aligned}
$$

we conclude that $\quad \widetilde{\omega}_{0}^{1}=1^{-1} U^{-1} \dot{h} \sin \theta d \phi=U^{-1} h^{-1} \dot{h} e^{1}$, which is the same as 
the one in the ambient space so that these cylinders are totally geodesic. Computing the curvature 2 -form,

$$
\begin{aligned}
\widetilde{R}_{1}^{0} & =d \widetilde{\omega}^{0}{ }_{1}+\widetilde{\omega}^{0} \wedge \widetilde{\omega}^{i}{ }_{1} \\
& =2^{-1}\left(U^{-2} \dot{U} h-U^{-1} \dot{h}\right) d r \wedge d \theta \\
& =\left(U^{-3} \dot{U}-U^{-2} h^{-1} \dot{h}\right) e^{0} \wedge e^{1} .
\end{aligned}
$$

So that the Gaussian curvature is equal to $\widetilde{R}_{0101}=\widetilde{R}^{0}{ }_{101}=U^{-3} \dot{U}-U^{-2} h^{-1} \dot{h}$.

$\mathbf{S}_{5}$ : Now, keep $\phi, \theta$ fixed, vary $r, \psi$ to obtain spheres. Metric restricted to these

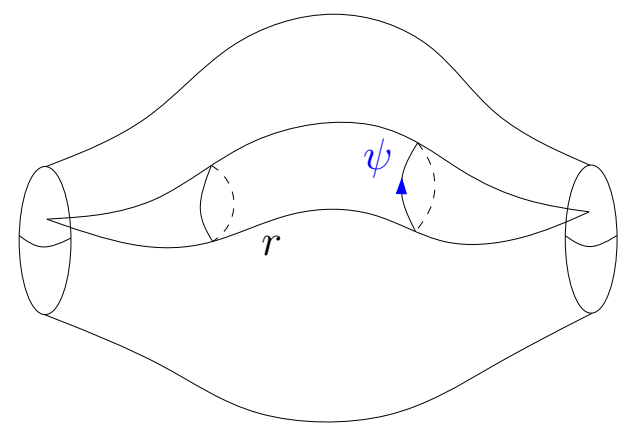

Figure 7: Varying $r$ and $\psi$.

spheres is computed as

$$
g_{S_{5}}=V d r^{2}+\frac{C \sin ^{2} r}{4 V(r)} d \psi^{2}
$$

and using the orthonormal coframe

$$
\left\{e^{0}, e^{2}\right\}=\left\{U d r, \frac{D \sin r}{2 U} d \psi\right\},
$$

we compute the connection 1-form,

$$
\widetilde{\omega}_{0}^{2}=\cos \theta d \phi=U^{-2}(-\dot{U}+U \cot r) e^{2}
$$

which is the same as the ambient one so that the second fundamental form vanishes and hence these spheres are totally geodesic and complex, see [KK18]. The curvature,

$$
\begin{gathered}
\widetilde{R}_{0}^{2}=d \widetilde{\omega}^{2}{ }_{0}+\widetilde{\omega}^{2}{ }_{i} \wedge \widetilde{\omega}^{i}{ }_{0} \\
=2^{-1} D U^{-3}\left\{3 \dot{U}\left(-U^{-1} \dot{U} \sin r+\cos r\right)+(\ddot{U} \sin r+U \sin r)\right\} d r \wedge d \psi . \\
\qquad \widetilde{R}_{0}^{2}=\frac{1}{2} \widetilde{R}^{2}{ }_{020} e^{2} \wedge e^{0}
\end{gathered}
$$


Then,

$$
\widetilde{R}^{2}{ }_{020}=U^{-3}\left\{3 \dot{U}\left(-U^{-1} \dot{U}+\cot r\right)+\ddot{U}+U\right\},
$$

and one can check through Gauss-Bonnet that,

$$
\int_{S_{5}} R_{020}^{2} d V=\int_{S_{5}} 2^{-1} D \sin r U^{-3}\left\{3 \dot{U}\left(-U^{-1} \dot{U}+\cot r\right)+\ddot{U}+U\right\} d r \wedge d \psi=4 \pi .
$$

$\mathbf{S}_{6}$ : We keep $r, \psi$ fixed and vary $\phi, \theta$ to obtain spheres, again. Then restricting the Page metric we get

$$
g_{S_{6}}=\left\{\frac{f}{4} \sin ^{2} \theta+\frac{C \sin ^{2} r \cos ^{2} \theta}{4 V(r)}\right\} d \phi^{2}+\frac{f}{4} d \theta^{2}
$$

and working with the orthonormal coframe $\left\{e^{1}, e^{3}\right\}$ we compute the connection 1-form,

$$
\widetilde{\omega}_{3}^{1}=\cos \theta d \phi=2 h^{-1} \cot \theta e^{1}
$$

is the same as the ambient one so that the second fundamental form vanishes and hence these spheres are totally geodesic. The curvature,

$$
\begin{gathered}
\widetilde{R}_{3}^{1}=d \widetilde{\omega}^{1}{ }_{3}+\widetilde{\omega}_{i}^{1} \wedge \widetilde{\omega}^{i}{ }_{3} \\
=-\sin \theta d \theta \wedge d \phi . \\
\widetilde{R}_{3}^{1}=\frac{1}{2} \widetilde{R}_{313}^{1} e^{1} \wedge e^{3} \\
\widetilde{R}_{313}^{1}=\frac{4}{f}=\text { const. }
\end{gathered}
$$

In particular we see that the spheres at the two endpoints $r=0$ or $\pi$ has constant positive curvature. These are the zero and infinity section of the Hirzebruch fibration or twisted sphere bundle map. Note that these two sections both have the same diameter since $f(0)=f(\pi)$. The general radius is $\operatorname{rad}(r)=1 / \kappa^{2}=$ $f^{2} / 16$.

We can summarize some of the properties we have observed as follows.

Theorem 1. All of the surfaces $S_{1} \cdots S_{6}$ are totally geodesic, hence minimal.

Theorem 2. We can list the constant Gaussian curvature surfaces in our list as follows,

1. The tori in the classes $S_{1}$ and $S_{3}$ are flat.

2. The spheres in the class $S_{6}$ are of constant positive curvature. 


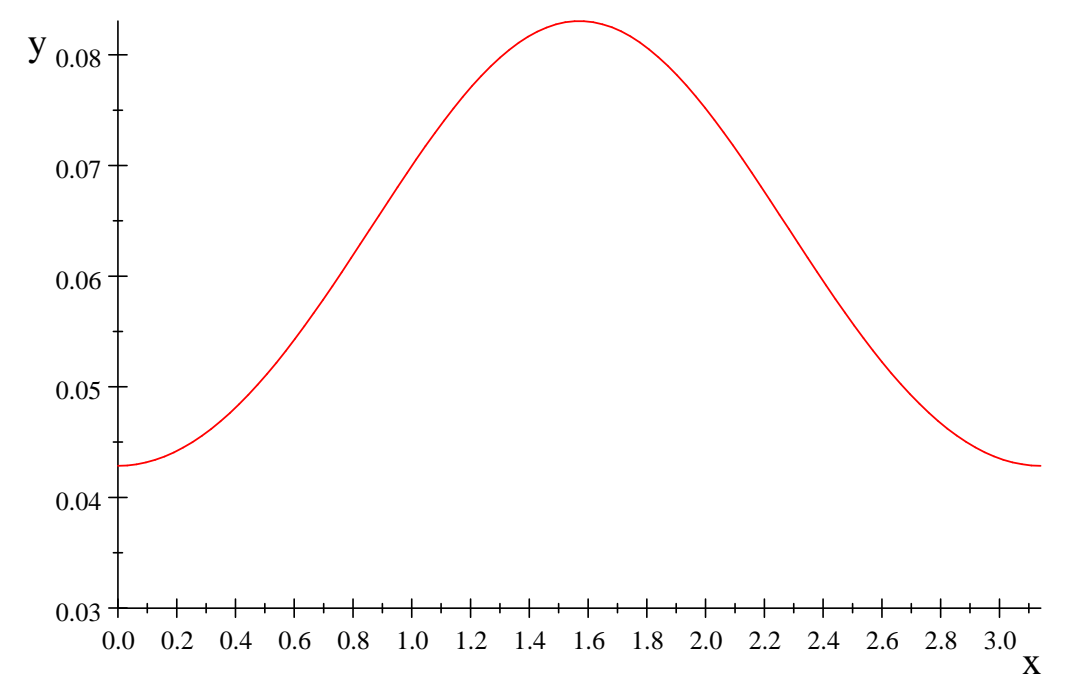

Figure 8: Radius function rad( $(r)$ for $\phi \theta$ spheres.

\section{Three dimensional minimal submanifolds}

In this section we will consider the 3 dimensional submanifold by fixing a variable in our coordinate system.

$\mathbf{N}_{1}$ : Easiest case is obtained when we fix the coordinate $r=r_{0} \in(0, \pi)$. This way we get totally geodesic 3-spheres endowed with Berger sphere metrics. At the endpoints $r_{0}=0, \pi$ this restriction gives round 2-spheres. From (1.1.1) we get

$$
\begin{gathered}
g_{N_{1}}:=f\left(r_{0}\right)\left(\sigma_{1}^{2}+\sigma_{2}^{2}\right)+\frac{C \sin ^{2} r_{0}}{V\left(r_{0}\right)} \sigma_{3}^{2} \\
=\widetilde{\sigma}_{1}^{2}+\widetilde{\sigma}_{2}^{2}+\frac{C \sin ^{2} r_{0}}{V\left(r_{0}\right)} \sigma_{3}^{2} . \\
\left|\sigma_{1}\right|=\left|\sigma_{2}\right|=1 / \sqrt{f\left(r_{0}\right)}, \quad\left|\sigma_{3}\right|=\frac{U}{D \sin r_{0}} .
\end{gathered}
$$

Then using the orthonormal coframe $\left\{e^{1}, e^{2}, e^{3}\right\}$ connection 1 -forms can be computed from the structure equations as follows,

$\widetilde{\omega}_{2}^{3}=D U^{-1} h^{-2} \sin r e^{1}, \widetilde{\omega}_{3}^{1}=2 h^{-1} \cot \theta e^{1}-D U^{-1} h^{-2} \sin r e^{2}, \widetilde{\omega}^{2}{ }_{1}=D U^{-1} h^{-2} \sin r e^{3}$

which are the same as the one $(\overline{1.5})$ of the ambient space, so that these type of submanifolds are totally geodesic and hence minimal. Computing the curvatures,

$$
\widetilde{R}_{212}^{1}=0, \quad \widetilde{R}_{313}^{1}=0
$$




$$
\widetilde{R}_{323}^{2}=4 h^{-2}=\text { const. }
$$

So these are constant scalar curvature but non-Einstein (i.e. non-constant sectional curvature in this dimension) spaces.

$\mathbf{N}_{2}$ : Keeping $\theta$ fixed we obtain the Seifert fibered 3-manifold $S^{1} \times S^{2}$. To see this note that $\psi, \phi$ traces a torus for $0<r<\pi$. At the endpoints the Hopf $\psi$ circles shrink. Looking in the $r, \psi$ direction we have an American football (i.e. the spheres of $S_{5}$ ), on which $\psi$ coordinates draw circles. This is the most direct way to see it. Alternatively, start with $I \times T^{2}$ which one can imagine as a thickened cylinder, whose inside is to be glued to its outside. Before gluings lets perform the shrinkings. At the endpoints, a set of circles shrink, so the top and bottom of the thickened cylinder must be shrank to circles. So this is a thickened cylinder but with sharpened ends. If we glue inside out, the neighborhood of these sharpened ends become solid tori. Now we have two solid tori glued along their boundary. Solid torus is the product of circle times disk $S^{1} \times D^{2}$, and when boundaries of two copies are identify, that means identifying the boundary of two disks which has to yield a sphere hence sphere times a circle. This solid tori decomposition is actually called the Heegard decomposition of this 3-manifold in the 3-manifold theory literature [Rol76]. A third way of interpretation can be done using the capping process which is described in detail in [AK12]. Shrinking a circle is the same operation as attaching a 2-disk which is called capping. Then it becomes easier to see the two solid tori at the end points. We have a $I \times T^{2}$, and only the two tori at the to endpoints of this manifold is filled to yield solid tori. The rest can be seen as gluing solid tori from a distance. The metric on this 3-manifold is,

$$
g_{N_{2}}=V d r^{2}+\left\{\frac{f}{4} \sin ^{2} \theta+\frac{C \sin ^{2} r \cos ^{2} \theta}{4 V(r)}\right\} d \phi^{2}+\frac{C \sin ^{2} r}{4 V(r)} d \psi^{2}+\frac{C \sin ^{2} r \cos \theta}{4 V(r)}(d \psi \otimes d \phi+d \phi \otimes d \psi) .
$$

Then working with the orthonormal coframe $\left\{e^{0}, e^{1}, e^{2}\right\}$ we conclude that

$$
\widetilde{\omega}_{0}^{1}=U^{-1} h^{-1} \dot{h} e^{1}, \quad \widetilde{\omega}_{0}^{2}=\left(U^{-1} \cot r-U^{-2} \dot{U}\right) e^{2} \text { and } \widetilde{\omega}_{2}^{1}=0,
$$

which is the same as the one in the ambient space so that this submanifold is totally geodesic. Computing the curvature 2-forms,

$$
\begin{gathered}
\widetilde{R}_{101}^{0}=2 h^{-1} U^{-3}(\ddot{h} U-\dot{h} \dot{U}), \\
\widetilde{R}_{202}^{0}=U^{-3}\{2 \dot{U}-U \ddot{U}-2 U \dot{U} \cot r-U\}, \quad \widetilde{R}^{1}{ }_{212}=0 .
\end{gathered}
$$

$\mathbf{N}_{3}$ : Keeping $\phi$ fixed we again obtain copies of $S^{1} \times S^{2}$ similar to the previous case. The metric is,

$$
g_{N_{3}}=V d r^{2}+\frac{C \sin ^{2} r \cos \theta}{4 V(r)}(d \psi \otimes d \phi+d \phi \otimes d \psi)+\frac{f}{4} d \theta^{2} .
$$


Then we have the orthonormal coframe

$$
\left\{e^{0}, e^{2}, e^{3}\right\}=\left\{U d r, \frac{D \sin r}{2 U} d \psi, \frac{h}{2} d \theta\right\},
$$

we conclude that

$$
\widetilde{\omega}_{0}^{2}=\left(U^{-1} \cot r-U^{-2} \dot{U}\right) e^{2}, \quad \widetilde{\omega}_{0}^{3}=0 \text { and } \widetilde{\omega}_{3}^{2}=0,
$$

which is the same as the one in the ambient space so that this submanifold is totally geodesic. Computing the curvatures,

$$
\begin{gathered}
\widetilde{R}_{020}^{2}=U^{-4}\left\{\dot{U} U+\dot{U} \cot r+2 \dot{U} U \cot r+U^{2}-3 \dot{U}^{2}\right\}, \\
\widetilde{R}_{303}^{0}=0, \quad \widetilde{R}_{323}^{2}=0 .
\end{gathered}
$$

$\mathbf{N}_{4}$ : Keeping $\psi$ fixed gives us a copy of the cylinder $S^{2} \times I$ with the following metric,

$$
g_{N_{4}}=V d r^{2}+\left\{\frac{f}{4} \sin ^{2} \theta+\frac{C \sin ^{2} r \cos ^{2} \theta}{4 V(r)}\right\} d \phi^{2}+\frac{f}{4} d \theta^{2} .
$$

Then using the orthonormal coframe $\left\{e^{0}, e^{2}, e^{3}\right\}$ we conclude that

$\widetilde{\omega}_{0}^{1}=U^{-1} h^{-1} \dot{h} e^{1}+2^{-1} U^{-1} h^{-1} \dot{h} \sin ^{-1} \theta e^{3}, \widetilde{\omega}_{0}^{3}=2^{-1} U^{-1} h^{-1} \dot{h} \sin ^{-1} \theta e^{1}, \widetilde{\omega}^{3}{ }_{1}=D U^{-1} h^{-2} \sin r e^{1}$

which is the same as the three in the ambient space so that this submanifold is totally geodesic. Computing the curvature,

$$
\begin{gathered}
\widetilde{R}^{0}{ }_{202}=-2 h^{-1} U^{-3}(\ddot{h} U-\dot{h} \dot{U}), \\
\widetilde{R}^{0}{ }_{303}=-h^{-1} U^{-3}(\ddot{h} U-\dot{h} \dot{U}), \quad \widetilde{R}_{323}^{2}=\dot{h}^{2} h^{-2} U^{-2} .
\end{gathered}
$$

\section{Relations with the Yamabe Problem and other met- rics on Hirzebruch surfaces}

We define the normalized Einstein-Hilbert functional on a smooth 4-manifold by,

$$
E(g)=v o l_{g}^{-1 / 2} \int_{M} R_{g} d V_{g} .
$$

Then for a conformal class $[g]=\left\{f g \mid f: M \rightarrow \mathbb{R}^{+}\right.$is $\left.C^{\infty}\right\}$ of a metric $g$, we define the Yamabe constant of this class by $Y_{[g]}:=\inf \left\{E\left(e^{f} g\right) \mid f\right.$ is $\left.C^{\infty}\right\}$. This infimum is shown to be achieved by a metric by Yamabe and we call the minimum as the Yamabe minimizer of the conformal class. As a result by Aubin, the space of Yamabe constants are bounded from above by the one of round spheres, so that 
$Y_{[g]} \leq E\left(S_{\text {Round }}^{4}\right)=24 \pi \sqrt{2 / 3} \approx 61.562393$. Consequently it has a supremum on a manifold $Y M(M)=\sup _{g \in \mathcal{M}} Y_{[g]}$ called the Yamabe Invariant of the smooth manifold. Here $\mathcal{M}$ denotes the space of all smooth metrics on the manifold $M$. We refer to the survey [Leb99] for details. The conjectural value of the Yamabe invariant of the Page manifold $\mathbb{C P}_{2} \sharp \overline{C P}_{2}$ is $12 \sqrt{2} \pi \approx 53.314598$ which is that of the complex projective plane by [LeB97]. Now, let's calculate the volume of Page metric. Using the coordinates $(1.2)$ we have,

$$
g_{i j}=\left(\begin{array}{cccc}
V & 0 & 0 & 0 \\
0 & \frac{f}{4} \sin ^{2} \theta+\frac{C \sin ^{2} r \cos ^{2} \theta}{4 V} & \frac{C \sin ^{2} r \cos \theta}{4 V} & 0 \\
0 & \frac{C \sin ^{2} r \cos \theta}{4 V} & \frac{C \sin ^{2} r}{4 V} & 0 \\
0 & 0 & 0 & \frac{f}{4}
\end{array}\right)
$$

and then,

$$
\operatorname{det} g_{i j}=\frac{C}{64} f^{2} \sin ^{2} r \sin ^{2} \theta
$$

then the volume form becomes,

$$
d V=\frac{D}{8} f(r) \sin r \sin \theta d r \wedge d \phi \wedge d \psi \wedge d \theta
$$

After all taking the variables $\theta \leq \pi$ and $\phi, \psi \leq 2 \pi$ we get the volume,

$$
\operatorname{Vol}_{\text {gpage }}=\int_{M} d V=\frac{16 \pi^{2}}{3\left(3+a^{2}\right)} \frac{3-a^{2}}{3+6 a^{2}-a^{4}} \approx 14.38828 .
$$

The scalar curvature of the Page metric can be computed to be $12\left(1+a^{2}\right) \approx 12.952$. So the Yamabe function takes the following value.

$$
E\left(g_{\text {Page }}\right)=12\left(1+a^{2}\right) v o l^{1 / 2} \approx 49.13 .
$$

Since this is an Einstein metric, by [Oba72] it is a Yamabe minimizer. So that this implies $Y M\left(\mathbb{C P}_{2} \sharp \overline{\mathbb{C P}}_{2}\right) \geq 49.13$. On the other hand, the Einstein-Hilbert action of an Otoba metric [Oto14] on the Page manifold with scalar curvature $R$ is given by

$$
E\left(g_{1}(R)\right)=2 \sqrt[4]{2} \pi R \sqrt{\operatorname{Arcsin}(k)}, \quad k^{2}=\left(1+\beta / \sqrt{2+\beta^{2}}\right) / 2, \quad \beta=(8-R) / 2 .
$$

This function indeed takes arbitrary small and large numbers for varying the values of $R$. Since Bach-flat metrics and Otoba metrics are mutually exclusive, Page metric is not in the set of Otoba metrics, neither in its conformal rescalings. Due to the theorem of Obata there is no other constant scalar curvature metric in the conformal class of an Einstein metric except for $S^{4}$. Instead, one of the Otoba metrics conformally equivalent to a metric in the Kähler class of the Page metric. 


\section{A Linear Structure Equations for Connection 1-forms}

In this appendix we are going to provide the solution to the system of differential equations providing the connection 1-forms. For the equation we are dealing with, the simplest particular solution techniques actually do not work. So one has to start with the most general form for the possibilities. That is the reason we decided to include it here. We start with writing down the structure equation system,

$$
\begin{aligned}
& 0=\quad 0 \quad+0+\omega_{1}^{0} \wedge e^{1}+\omega^{0}{ }_{2} \wedge e^{2}+\omega^{0}{ }_{3} \wedge e^{3} \\
& 0=\quad U^{-1} h^{-1} \dot{h} e^{01}+2 h^{-1} \cot \theta e^{31} \quad+\omega_{0}^{1} \wedge e^{0}+0+\omega^{1}{ }_{2} \wedge e^{2}+\omega_{3}^{1} \wedge e^{3} \\
& 0=-\sin ^{-1} r\left(U^{-1} \sin r\right)_{r} e^{20}-2 D U^{-1} h^{-2} \dot{h}^{-1} \sin r e^{31}+\omega^{2}{ }_{0} \wedge e^{0}+\omega^{2}{ }_{1} \wedge e^{1}+\quad 0+\omega^{2}{ }_{3} \wedge e^{3} \\
& 0= \\
& U^{-1} h^{-1} \dot{h} \sin ^{-1} \theta e^{01} \\
& +\omega^{3}{ }_{0} \wedge e^{0}+\omega^{3}{ }_{1} \wedge e^{1}+\omega^{3}{ }_{2} \wedge e^{2}+0 .
\end{aligned}
$$

which involves a system of 4 equations and 6 unknowns. Using (0) we label the first three forms as follows.

$$
\omega_{0}^{1}=\alpha e^{1}+\beta e^{2}+\gamma e^{3}, \omega_{0}^{2}=\beta e^{1}+\varepsilon e^{2}+\delta e^{3}, \quad \omega_{0}^{3}=\gamma e^{1}+\delta e^{2}+\theta e^{3} .
$$

Imposing each of them, the related other equation $(1,2,3)$ we reach,

$$
\omega_{0}^{1}=U^{-1} h^{-1} \dot{h} e^{1}+\beta e^{2}+\gamma e^{3}, \omega^{2}{ }_{0}=\beta e^{1}+\sin ^{-1} r\left(U^{-1} \sin r\right)_{r} e^{2}+\delta e^{3}, \omega_{0}^{3}=\gamma e^{1}+\delta e^{2} .
$$

Using $(3,2)$ we start with the form $\omega^{3}{ }_{2}=-\delta e^{0}+\rho e^{1}$ and $\omega^{1}{ }_{2}=\sigma e^{0}+\tau e^{3}$ using (2). Next, imposing (3) we start with the following form,

$$
\omega^{3}{ }_{1}=\left(\gamma-U^{-1} h^{-1} \dot{h} \sin ^{-1} \theta\right) e^{0}+\zeta e^{1}+\rho e^{2} .
$$

We will work on equation (1). Matching the 03-terms we can compute $\gamma=$ $2^{-1} U^{-1} h^{-1} \dot{h} \sin ^{-1} \theta$. Comparing the 13 -terms we get $\zeta=-2 h^{-1} \cot \theta$ and comparing 23-terms we get $\rho=-\tau$. We continue comparisons within the same equation by 02 -terms to get $\sigma=\beta$. Comparison within the next equation (2) yields $\beta=0$ and $\tau=-D U^{-1} h^{-2} \sin r$. Collecting these we able to settle the forms,

$$
\begin{gathered}
\omega^{3}{ }_{1}=-2^{-1} U^{-1} h^{-1} \dot{h} \sin ^{-1} \theta e^{0}+-2 h^{-1} \cot \theta e^{1}+D U^{-1} h^{-2} \sin r e^{2}, \\
\omega^{1}{ }_{2}=-D U^{-1} h^{-2} \sin r e^{3} .
\end{gathered}
$$

To figure out $\delta$ we consult to the final equation (3). Comparing the 02-terms here yields $\delta=0$. 


\section{References}

[AK12] Selman Akbulut and Mustafa Kalafat. A class of locally conformally flat 4-manifolds. New York J. Math., 18:733-763, 2012.

[EGH8o] Tohru Eguchi, Peter B. Gilkey, and Andrew J. Hanson. Gravitation, gauge theories and differential geometry. Phys. Rep., 66(6):213-393, 1980.

[FIPo4] Maria Falcitelli, Stere Ianus, and Anna Maria Pastore. Riemannian submersions and related topics. World Scientific Publishing Co., Inc., River Edge, NJ, 2004.

[Joso6] Jürgen Jost. Compact Riemann surfaces. Universitext. Springer-Verlag, Berlin, third edition, 2006. An introduction to contemporary mathematics.

[KK18] Mustafa Kalafat and Caner Koca. On the curvature of EinsteinHermitian surfaces. Illinois J. Math., To appear, 2018.

[KR88] Brian W. Kernighan and Dennis M. Ritchie. The C Programming Language. Prentice Hall Professional Technical Reference, 2nd edition, 1988.

[LeB97] Claude LeBrun. Yamabe constants and the perturbed Seiberg-Witten equations. Comm. Anal. Geom., 5(3):535-553, 1997.

[Leb99] Claude Lebrun. Einstein metrics and the Yamabe problem. In Trends in mathematical physics (Knoxville, TN, 1998), volume 13 of AMS/IP Stud. Adv. Math., pages 353-376. Amer. Math. Soc., Providence, RI, 1999.

[Oba72] Morio Obata. The conjectures on conformal transformations of Riemannian manifolds. J. Differential Geometry, 6:247-258, 1971/72.

[Oto14] Nobuhiko Otoba. Constant scalar curvature metrics on Hirzebruch surfaces. Ann. Global Anal. Geom., 46(3):197-223, 2014.

[Pag78] D. Page. A Compact Rotating Gravitational Instanton. Phys. Let., 79B(3):235-238, 1978 .

[Rol76] Dale Rolfsen. Knots and links. Publish or Perish, Inc., Berkeley, Calif., 1976. Mathematics Lecture Series, No. 7 .

Orta mh. ZÜbeyde Hanim cd. No 5-3 Merkez 74100 Bartin, TÜrKíYe.

E-mail address: kalafat@ math.msu.edu

Amasya Üníversítesí, Merzífon Myo, 05300, TÜRKÍYe.

E-mail address: ramazan.sari@ amasya.edu.tr 